\title{
Behavior of Ionic Conduction in Poly(methyl methacrylate) under a High Electric Field
}

\author{
Teruo Miyamoto \\ Ako Plant, Mitsubishi Electric Corporation, \\ Ako, Hyogo, Japan.
}

(Received January 21, 1974)

\begin{abstract}
Electrical conduction of poly(methyl methacrylate) (PMMA) is considered to be ionic from low to very high fields near breakdown. Support for the ionic mechanism can be supplied from the compensation law, and from the temperature dependence of the electrical conductivity and the breakdown field. In high electric fields above the ohmic region, it was observed that the apparent activation energy decreased with increasing electric field. A simplified equation for the activation energy was derived in which the dependence of the electric field on ionic jump energy and ionic dissociation energy was taken into consideration. The ionic jump distance between equilibrium positions computed from the equation was 10 to $20 \AA$ and was in good agreement with periodic structure of PMMA.
\end{abstract}

KEY WORDS Electrical Conduction / Ionic Conduction / Poly(methyl methacrylate) / Ionic Jump Distance / Ionic Dissociation Energy /

Even an insulating material such as an organic polymer possesses a fairly accurately detectable current density. It is, however, sometimes not clear whether the charge-transfer process for a particular polymer involves the migration of ions or of electrons. Many authors ${ }^{1-4}$ suggest that most polymers except polymers with conjugated double bonds show an ionic conduction in relatively low strength fields. On the other hand, in high strength fields, even in polymers without conjugated double bonds, many experimental results ${ }^{5-7}$ were found to be favorable to the hypothesis of electronic conduction. The problem of electrical conduction in polymers in high field is so complicated that many processes not yet fully explained are involved.

The purpose of this investigation is to elucidate the electrical conduction behavior in poly(methyl methacrylate) under high field conditions.

\section{EXPERIMENTAL}

\section{Material}

The poly(methyl methacrylate) used for measurement was prepared by polymerization of distilled monomer using $\alpha, \alpha^{\prime}$-azobisisobutyro- nitrile as an initiator. The liquid monomer was polymerized by heating at $80^{\circ} \mathrm{C}$ for $4 \mathrm{hr}$, then at $120^{\circ} \mathrm{C}$ for $4 \mathrm{hr}$, between two glass plates, and finally at $160^{\circ} \mathrm{C}$ for $4 \mathrm{hr}$ in a vacuum.

\section{Measurements}

A film specimen was cut to about $50 \times 50 \mathrm{~mm}$. An electrode with guard-ring was applied to the test film by evaporating aluminum. The high voltage dc source used for the measurement of high field conduction could supply up to 15 $\mathrm{kV}$ and that for breakdown measurement could supply up to $70 \mathrm{kV}$. Dc conductivity was measured using a vibrating reed electrometer (Takeda Riken Co., TR-84M) in such a way as to evaluate it from steady-state current observed after eliminating effects due to absorbed charge when the specimen was charged with electricity. The breakdown test was conducted by a raising the voltage at a rate of $1 \mathrm{kV} / \mathrm{sec}$.

Dielectric properties were determined with a mutual inductance bridge (Ando Electric Co., TR-10C) in the temperature region from room temperature to $160^{\circ} \mathrm{C}$.

The temperature dependence of specific volume was measured from the variation in weight with temperature in a silicone oil medium by using 


\section{T. Miyamoto}

the direct weighing balance modified for the measurement of specific volume.

\section{RESULTS}

Plots of logarithmic electrical conductivity vs. reciprocal absolute temperature under various electric field strengths are shown in Figure 1. Values of the electrical conductivity in relatively low electric field strength coincide well with each other and increase with electric field strength in the considerably higher electric field region. From the electric field dependence of the electric current density at $63^{\circ} \mathrm{C}$ and $113^{\circ} \mathrm{C}$, the ohmic law holds up to an electric field of approximately $0.1 \mathrm{MV} / \mathrm{cm}$. The slope in Figure 1 changes at a critical temperature. It is observed that the critical temperature in the nonohmic region shifts toward a lower temperature as the electric field increases. The electrical conductivity $\sigma$ in Figure 1 is expressed by following the Arrhenius equation

$$
\sigma=\sigma_{0} \exp \left(-\frac{E_{\mathrm{A}}}{R T}\right)
$$

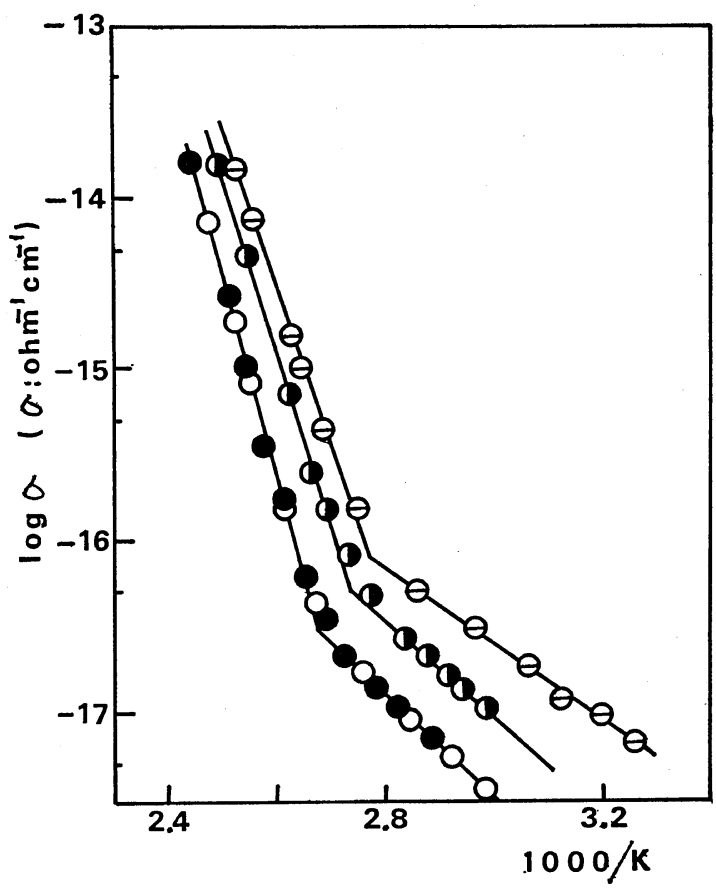

Figure 1. Electrical conductivity vs. reciprocal absolute temperature: $\bigcirc, 0.014 \mathrm{MV} / \mathrm{cm} ; 0,0.086$ $\mathrm{MV} / \mathrm{cm}$;, $0.36 \mathrm{MV} / \mathrm{cm} ; \ominus, 0.74 \mathrm{MV} / \mathrm{cm}$. where $\sigma_{0}$ is a constant, $E_{\mathrm{A}}$ is the Arrhenius activation energy, $R$ is the gas constant, and $T$ is the absolute temperature. The apparent activation energy of a linear polymer obtained by such Arrhenius plots is in most cases $20-45 \mathrm{kcal} /$ mol at a temperature above the glass transition temperature $^{8}$ and the values of the apparent activation energy obtained here fall within this range. The apparent activation energy, however, tends to decrease as the electric field strength increases.

The temperature dependence of specific volume is shown in Figure 2. Intersection of oblique lines shows the glass transition temperature $\left(104^{\circ} \mathrm{C}\right)$.

The temperature dependence of the breakdown field is shown in Figure 3. The intersection of the oblique lines was obtained by the method of least squares and the intersection can be

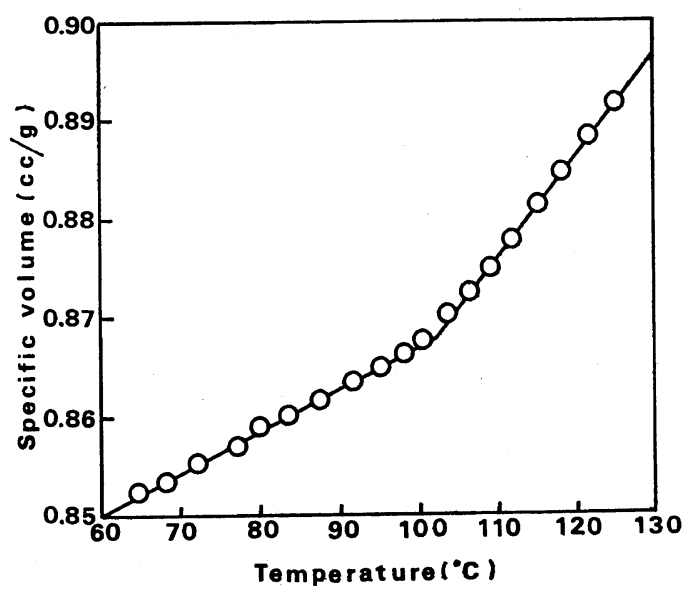

Figure 2. Specific volume vs. temperature.

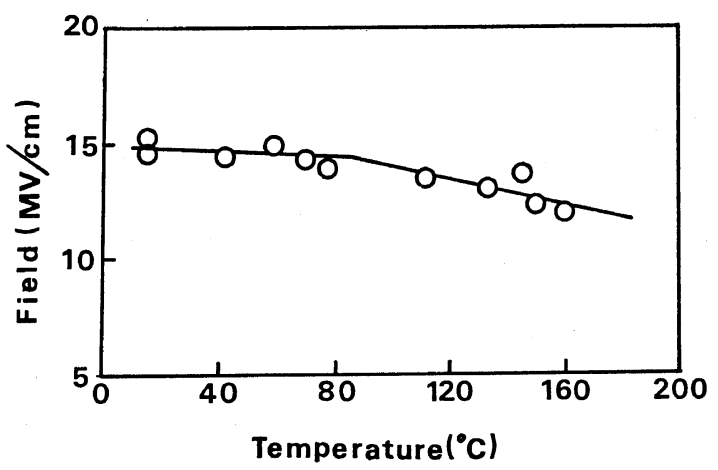

Figure 3. Breakdown field vs. temperature.

Polymer J., Vol. 6, No. 5, 1974 
Ionic Conduction in Poly(methyl methacrylate)

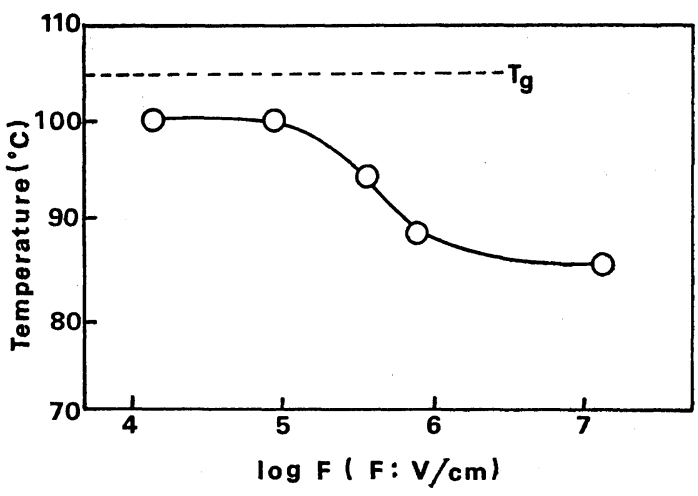

Figure 4. Critical temperature obtained from Figure 1 vs. electric field strength.

related to the glass transition, although experimental data scatter considerably. The critical temperature obtained from Figure 1 is located between the glass transition temperature $\left(104^{\circ} \mathrm{C}\right)$ and the temperature $\left(85^{\circ} \mathrm{C}\right)$ of the intersection of the oblique lines in Figure 3 as shown in Figure 4.

\section{DISCUSSION}

The preexponential term and the apparent activation energy correlate closely with each other. Large values of activation energy are often found to be associated with large values of the preexponential term, and the logarithm of the preexponential term is found to increase linearly with increasing activation energy. This relationship has been termed a compensation law and has been observed for transport phenomena such as rate of chemical reaction, viscosity, diffusion and so on.

The compensation law has been reported not only in these transport phenomena but also in the electrical conduction behavior of polymers with variation of the structural features: indophenine polymers ${ }^{9}$ or epoxy resins ${ }^{10}$ with various structures and poly(ethylene terephthalate) with change of crystallinity ${ }^{4}$. As shown in Figure 5 where the data have been collected from review articles ${ }^{11-14}$ and the literature ${ }^{9,15,16}$, the compensation law appears to hold for electrical conduction in many polymers and the plots of the logarithm of the preexponential term vs. the apparent activation energy are divided into two distinct groups A and B. The conduction processes in indophenine polymers and other semiconducting polymers belong to group $\mathrm{A}$ and the data for ion-conducting polymers $^{2}$ are located on the line of group B. It is therefore plausible to expect that the compensation law can be a criterion to judge whether the mechanism of electrical conduction is electronic or ionic. The compensation law in

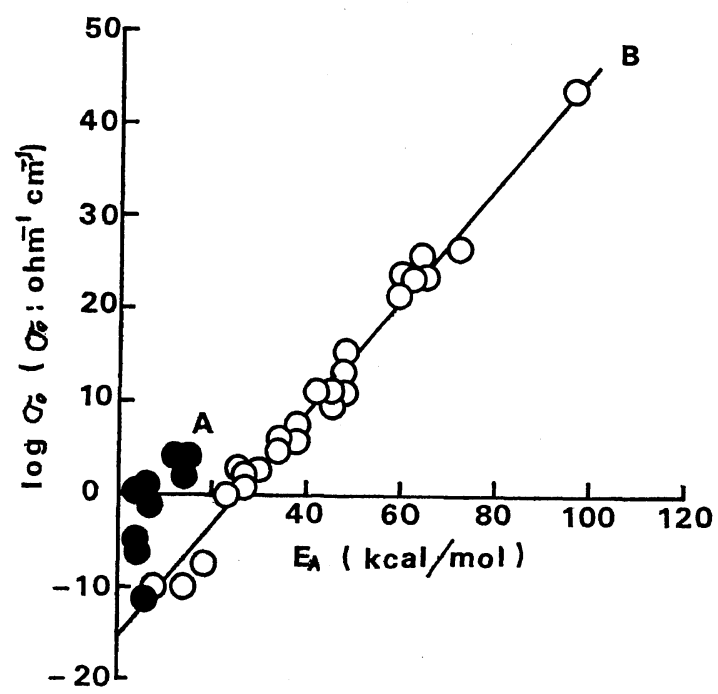

Figure 5. Compensation law of electrical conduction in polymers: $\bigcirc$, electronic conduction; $\bigcirc$, ionic conduction.

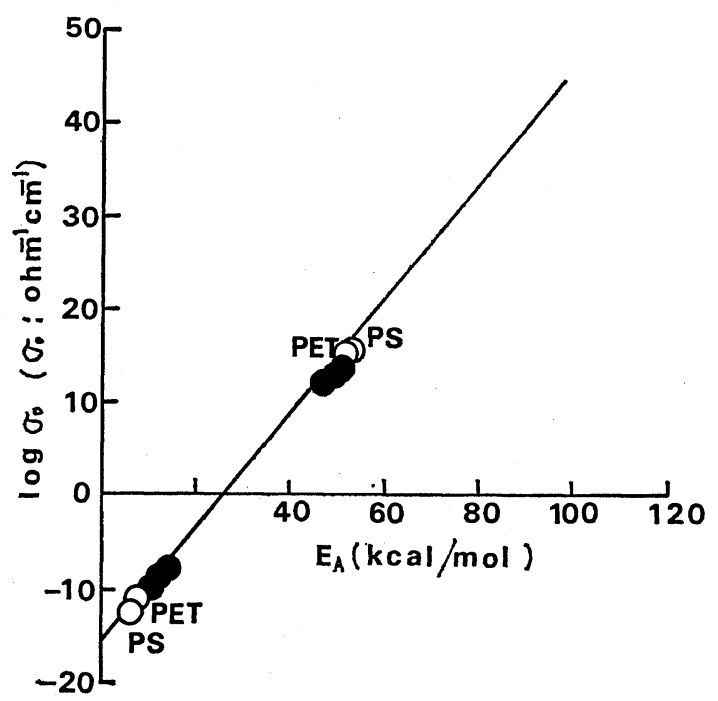

Figure 6. Compensation law in PMMA: PMMA. 
PMMA measured by varing the electric field strength is expressed by the straight line of group $B$ as shown in Figure 6 . In addition to this analysis, the marked change in slope of the temperature dependence of electrical conductivity plots in the vicinity of the glass transition temperature $T_{\mathrm{g}}$ is also favorable to the hypothesis of ionic conduction. The marked changes in slope is related to phenomena that microBrownian motion of polymer chains becomes active in the amorphous region. This motion is hindered by potential energy barriers at temperatures below $T_{\mathrm{g}}$. The energy becomes great enough to overcome these barriers with increasing temperature and segmental motion results in an increase in the free volume. Since the distance between molecules is a decisive factor for the migration of electrons, increase in the free volume or in irregularity at $T_{\mathrm{g}}$ would hinder the migration of electrons. Besides, the temperature dependence of the breakdown field in Figure 3 was fitted by two straight lines and the intersection also occurs at the glass transition region. This suggests that ionic conduction holds until very close to the breakdown field. It seems therefore that the breakdown phenomenon takes place abruptly over a very narrow range of electric field. On the other hand, Figure 6 also shows the compensation law of poly(ethylene terephthalate) $(\mathrm{PET})^{17}$ and polystyrene (PS) ${ }^{17}$ for reference.

It was observed that the critical temperature in the Arrhenius plots shifted toward a lower temperature with increasing the applied electric field strength. As one interpretation, the results of Figure 1 seem to be brought about by Joule's heat generated under applied high electric field. In the case of low applied electric field, the generation of Joule's heat may be negligibly small because of radiation. On the other hand, in a previous paper, ${ }^{18}$ the reason that the critical temperature does not always coincide with the glass transition temperature was explained by difference in the size of ionic charge carrier and segment.

The Arrhenius activation energy $E_{\mathrm{A}}$ taking the theory of ionic dissociation into consideration is derived by the electric field $F$ dependence as follows ${ }^{17}$

$$
E_{\mathrm{A}}(F)=E_{\mathrm{h}}(F)+E_{\mathrm{j}}(F)+\frac{1}{2} W(F)
$$

where $E_{\mathrm{h}}$ is the critical hole formation energy required for migration of ionic charge carrier, $E_{\mathrm{j}}$ is the potential barrier between equilibrium positions, and $W$ is the ionic dissociation energy. When $E_{\mathrm{h}}$ has the electric field dependence, it may be necessary to consider Maxwell stress which affects mainly the free volume. In the previous paper, it has been shown that $E_{\mathrm{h}}$ is expressed as the difference in the Arrhenius activation energy at temperatures above and below $T_{\mathrm{g}}{ }^{17}$ Values of $E_{\mathrm{h}}$ were found to be approximately $36 \mathrm{kcal} / \mathrm{mol}$ here irrespective of the applied field. This result suggests that the Maxwell stress has no appreciable effect. The electric field strength dependence of the Arrhenius activation energy, therefore, is considered to be brought about by change of potential barrier between equilibrium positions and the ionic dissociation energy. However, the effect of field strength on ionic dissociation was suggested to be negative by some authors. ${ }^{19,20}$ If the electric field dependence of ionic dissociation is allowed, $W(F)$ is expressed according to Onsager's law $^{21}$ as follows

$$
W(F)=W-2 e\left(\frac{e}{\varepsilon}\right)^{1 / 2} \sqrt{F}
$$

where $W$ is the ionic dissociation energy without electric field, $e$ is the ionic charge, and $\varepsilon$ is the dielectric constant. The ionic dissociation occurs due to thermal energy even when the field is absent. To get the condition of $2 e(e / \varepsilon)^{1 / 2} \sqrt{F}>k T$, $F$ is required to become higher than $0.1 \mathrm{MV} /$ $\mathrm{cm}$.

On the other hand, $E_{\mathrm{j}}(F)$ is expressed by the hopping model ${ }^{22,23}$. According to this model high field such as $e \lambda F \gg k T$ can be assumed to be negligible chance of jumping in the backward direction of applied field, where $\lambda$ is the jump distance. Namely $E_{\mathrm{j}}(F)$ in high field is expressed by the form

$$
E_{\mathrm{j}}(F)=E_{\mathrm{j}}-\frac{e \lambda F}{2}
$$

where $E_{\mathrm{j}}$ is the potential barrier without electric field. Consequently, the electric current density $i$ and $\Delta E_{\mathrm{A}}$ denoting the difference in 
$E_{\mathrm{A}}(F)$ and $E_{\mathrm{A}}(0)$ are expressed by combining eq 3 and 4

$$
\begin{aligned}
& i=C \exp \left\{\frac{e \lambda}{2 k T} F+\frac{e(e / \varepsilon)^{1 / 2}}{k T} \sqrt{F}\right\} \\
& \Delta E_{\mathrm{A}}=\frac{e \lambda}{2} F+e\left(\frac{e}{\varepsilon}\right)^{1 / 2} \sqrt{F}
\end{aligned}
$$

where $C$ is a constant. To apply the eq 5 for experimental data on the electric field strength dependence of current density, correction of temperature is necessary because the critical temperature in the Arrhenius plots has been observed to shift toward a lower temperature with the applied electric field strength. Therefore, the ionic jump distance $\lambda$ was estimated here from eq 6. We used the static dielectric constant obtained from the extrapolation of the Cole-Cole arc to the abscissa. The temperature dependence of ionic jump distance is shown in Figure 7. The noticeable fact is that the ionic jump distance changes somewhat at the glass transition region. This change is mainly due to the increase in the static dielectric constant at the glass transition region explained by the liberation of micro-Brownian motion of the main polymer chain. Although detailed information about the higher-order structure of polymer is lacking here, increase in the free volume or in irregularity of the main polymer chain would increase the distance between equilibrium positions. Kosaki, et al., ${ }^{24}$ have also observed recently similar behavior by analyzing the hyperbolic sine law in poly(vinyl chloride). At any rate, these observations suggest that the jump

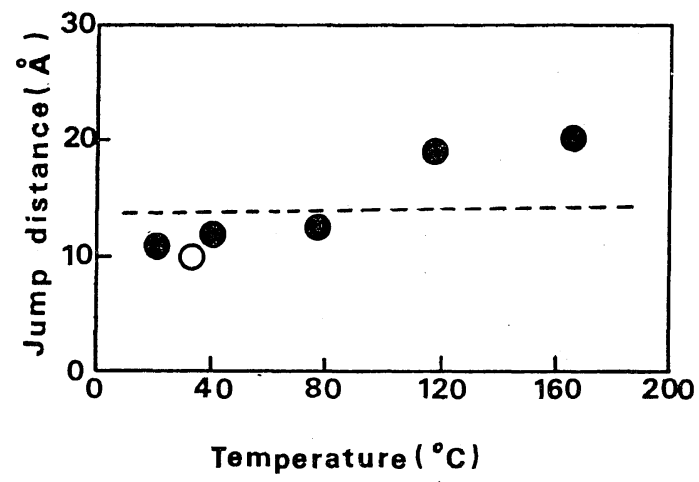

Figure 7. Jump distance vs. temperature: jump distance; $\bigcirc$, mean free path of phonon; dotted line, diameter of monomer unit. distance is correlated closely with structure or segmental motion of polymer.

If the monomer unit of polymer is regarded as a sphere, the size of the sphere can be computed from the specific volume of polymer. As shown by the dotted line in Figure 7, the diameter of the monomer unit is approximately $14 \AA$ at temperatures above and below the glass transition region and coincides well with the jump distance estimated here. Further, the mean free path of phonon in thermal conduction was estimated to be $10 \AA$ at $300^{\circ} \mathrm{K}$ by Wada. ${ }^{25}$ It is, therefore, said that periodic structure of PMMA is approximately $10 \AA$ at glassy region. Since the ratio of the critical hole required for migration of the ionic charge carrier to that for segmental motion is quite large (value about one third ${ }^{17}$ ), it can be easily recognized that the migration of ion is strongly regulated by the structure of the polymer.

\section{REFERENCES}

1. E. J. Murphy, Can. J. Phys., 41, 1022 (1963).

2. S. Saito, H. Sasabe, T. Nakajima, and K. Yada, J. Polym. Sci., Part A-2, 6, 1297 (1968).

3. T. Miyamoto and K. Shibayama, Kobunshi Kagaku (Chem. High Polymers), 29, 301 (1971).

4. B. I. Zazhin and N. G. Podesenova, Soviet Phys. Solid State, 6, 1755 (1965).

5. J. F. Fowler, Proc. Roy. Soc., Ser. A, 236, 464 (1956).

6. K. Yahagi and K. Shinohara, J. Appl. Phys., 37, 310 (1966).

7. A. van Roggen, Phys. Rev. Lett., 9, 363 (1962).

8. M. Tsunoda, H. Kawai, and M. Saito, "Structure and Properties of Polymers', Maruzen, Tokyo, 1963, p 347.

9. I. Schopov and C. Vodenicharov, J. Macromol. Sci.-Chem., A4, 1627 (1970).

10. R. W. Warfield and M. C. Petree, Macromol. Chem., 58, 139 (1962).

11. S. Hirota, Reports Electrotech. Lab., 1969, No. 166.

12. L. V. Gregor, IBM. J., 1968, p 140.

13. J.E. Katon, "Organic Semiconducting Polymers', Marcel Dekker Inc., New York, N.Y., 1970.

14. A. Amamiya and T. Watanabe, Oyo Butsuri, 26, 293 (1957).

15. W. Kloffer and H. Robenhorst, J. Chem. Phys., 49, 156 (1968). 
16. R. Somoano, S. P.S. Yen, and A. Rembaum, Polym. Lett., 8, 467 (1970).

17. T. Miyamoto and K. Shibayam, Kobunshi Kagaku (Chem. High Polymers), 30, 103 (1973).

18. T. Miyamoto and K. Shibayama, Polymer J., 6, 79 (1974).

19. R. A. Foss and W. Dannhavser, J. Appl. Polym. Sci., 7, 1015 (1963).

20. J. R. Price, Ann. Rep. C.E.I.D.P., 1967, p 32.

21. L. Onsager, J. Chem. Phys., 2, 599 (1934).
22. S. Glasstone, K. J. Laider, and H. Eyring, "The Theory of Rate Processes", McGrawHill, New York, N.Y., 1941, p 552.

23. R. E. Barker Jr., and C. R. Thomas, J. Appl. Phys., 35, 3203 (1964).

24. M. Kosaki, K. Sugiyama, and M. Ieda, ibid., 42, 3388 (1971).

25. Y. Wada, Preprints SPSJ 15th Symposium on Macromolecules, Osaka, Nov. 1966, p 125. 